\title{
Dynamic-Symmetry-Breaking Breathing and Spreading Transitions in Ferromagnetic Film Irradiated by Spherical Electromagnetic Wave
}

\author{
Muktish Acharyya \\ Department of Physics, Presidency University \\ 86/1 College Street, Calcutta-700073, India. \\ E-mail:muktish.physics@presiuniv.ac.in
}

The dynamical responses of a ferromagnetic film to a propagating spherical electromagnetic wave passing through it are studied by Monte Carlo simulation of two dimensional Ising ferromagnet. For a fixed set of values of the frequency and wavelength of the spherical EM wave, and depending on the values of amplitude of the EM wave and temperature of the system, three different modes are identified. The static pinned mode, the localised dynamical breathing mode and extended dynamical spreading mode are observed. The nonequilibrium dynamical-symmetry-breaking breathing and spreading phase transitions are also observed and the transition temperatures are obtained as functions of the amplitude of the magnetic field of EM wave. A comprehensive phase diagram is drawn. The boundaries of breathing and spreading transitions merge eventually at the equilibrium transition temperature for two dimensional Ising ferromagnet as the value of the amplitude of the magnetic field becomes vanishingly small.

PACS Nos:05.50.+q, 05.70.Ln, 75.30.Ds, 75.30.Kz, 75.40.Gb Keywords: Ising ferromagnet, Monte Carlo simulation, Spherical Electromagnetic wave, Symmetry breaking, Dynamical phase transition 


\section{Introduction}

Ising model is a widely used prototype to study the phase transitions phenomena. Because of its simplicity, even in the case of nonequilibrium behaviour, this model is being extensively and successfully used[1]. The hysteresis and dynamical phase transitions are two important responses of kinetic Ising ferromagnet to an oscillating magnetic field and play an important role in modern research of nonequilibrium phenomena[1].

Particularly, in this field, the journey was started to study the responses of kinetic Ising model to an oscillating magnetic field. The dynamical meanfield equation was solved and dynamic phase transition was observed [2]. Much effort was devoted to study the responses by Monte Carlo simulations. The hysteretic responses and dynamic symmetry breaking nonequilibrium phase transition were studied extensively. A considerable amount of research was performed to establish this phase transition as a nonequilibrium phase transition $[3,4,5,6,7,8,9,10,11]$. Recently, the surface and bulk dynamic transitions were studied in kinetic Ising model and the different classes of universality were observed[12].

Not only in Ising ferromagnet, the dynamic phase transition was observed in Ising metamagnet both from meanfield study[13] and Monte Carlo simulations[14]. The various kinds of nonequilibrium phase transitions were observed in classical vector spin models. Recently, the nonequilibrium phase transition was observed[15] in magnetic nanocomposites by $\mathrm{MC}$ simulations.

Apart from the simulational studies of kinetic Ising model, researchers are also paying attention to observe the dynamical phase transitions in BlumeCapel model[16], Blume-Emery-griffith model[17] and classical vector spin models[18, 19, 20, 21].

Experimentally, the dynamic symmetry breaking was also observed in ultrathin $\mathrm{Co}$ film on $\mathrm{Cu}(001)$ by surface magneto-optic Kerr effect[22] . However, it may be mentioned here that all the studies referred above have a common feature. In those cases, the magnetic field was sinusoidally oscillating but was uniform over the space at any particular instant.

Very recently, the nonequilibrium dynamic phase transition was also observed in Ising ferromagnet swept by linearly polarised electromagnetic plane wave[23, 24, 25]. In these cases, the spatio-temporal variations of the magnetic field were considered. Here, the coherent motion of spin clusters was found and phase boundaries were drawn.

In this present article, the dynamical responses of two dimensional Ising 
ferromagnet to a electromagnetic spherical wave are studied by Monte Carlo simulation. The layout of the paper is as follows: section-II decribes the model and the Monte Carlo simulation scheme, the numerical results are reported in section-III, the paper ends with concluding remarks in sectionIV.

\section{Model and simulation}

The two dimensional Ising ferromagnet (having uniform nearest neighbour interaction) in presence of a propagating spherical electromagnetic field wave (having spatio-temporal variation) can be represented by the following time dependent Hamiltonian,

$$
H(t)=-J \Sigma s(x, y, t) s\left(x^{\prime}, y^{\prime}, t\right)-\Sigma h(x, y, t) s(x, y, t)
$$

The Ising spin variable, $s(x, y, t)$ assumes value \pm 1 at lattice site $(x, y)$ at time $t$ on a square lattice of linear size $L$. The uniform ferromagnetic nearest neighbour interaction strength is $J(>0)$. The first sum represents the Ising spin-spin interaction. The spin-field interaction resides in the second summation. The $h(x, y, t)$ is the value of the magnetic field (at point $(x, y)$ and at any time $t$ ) of the propagating (radially outward) spherical electromagnetic wave (originating from the centre $\left(x_{0}, y_{0}\right)$ of the lattice). The form of spherically propagating wave is

$$
h(x, y, t)=h_{0} \frac{\mathrm{e}^{i\left(2 \pi f_{0} t-2 \pi r / \lambda\right)}}{r}
$$

Where, $r=\sqrt{\left(x-x_{0}\right)^{2}+\left(y-y_{0}\right)^{2}}$. The $h_{0}, f_{0}=\frac{\omega_{0}}{2 \pi}$ and $\lambda$ represent the amplitude, frequency and the wavelength respectively of the propagating spherical electromagnetic field wave which originates from $r=0$ and propagates radially outwards. This form of the propagating field was obtained from the solution of spherically symmetric Maxwell's equation representing the electromagnetic wave. In the present simulation, a $L \times L$ square lattice is considered. The boundary condition, used here, is periodic in both $(x$ and $y$ ) directions. The initial $(t=0)$ configuration, as the all spins are up $(s(x, y, t=0)=+1$ for all $x$ and $y))$, is taken here. The spins are updated randomly (a site $(x, y)$ is chosen at random) and spin flip occurs (at temperature $T$ ) according to the Metropolis probability[26] of single spin flip 
$(W)$

$$
W(s \rightarrow-s)=\operatorname{Min}[\exp (-\Delta E / k T), 1],
$$

where $\Delta E$ is the change in energy due to spin flip and $k$ is Boltzmann constant. $L^{2}$ such random updates of spins defines the unit time step here and is called Monte Carlo Step per spin (MCSS). Here, the magnetic field and the temperature are measured in the units of $J$ and $J / k$ respectively. The dynamical steady state is reached by heating the system slowly (in presence of the propagating field) in small step ( $\delta T=0.05$ here) of temperature. It may be mentioned here that the same dynamical steady state was observed to be achieved by cooling the system from a high temperature random configuration. The frequency and wavelength of the propagating magnetic field were kept fixed $(f=0.01$ and $\lambda=15.0)$ throught the study. The total length of simulation is $2 \times 10^{5} \mathrm{MCS}$ and first $10^{5} \mathrm{MCS}$ transient data were discarded to achieve the stable dynamical steady state. Since the frequency of the propagating field is $f=0.01$, the complete cycle of the field requires 100 MCS. So, in $10^{5}$ MCS, $10^{3}$ numbers of cycles of the propagating field are present. The time averaged data over the full cycle (100 MCSS) of the propagating field are further averaged over 1000 cycles. Here, the number of cycles is denoted by $n_{c}$.

The quantities measured are: Instantaneous local magnetisation density in the circle of radius $\lambda / 2$ is $m_{b}(t)=\sum s(x, y, t) /\left(N_{b}\right)$, where the sum is carried over the number of sites $\left(N_{b}\right)$ lying within the circle of radius $\lambda / 2$ centered at the centre $\left(x_{0}, y_{0}\right)$ of lattice. In the present study, the lattice size $L$ is taken equal to 101. So, the coordinates of the centre is $\left(x_{0}=51, y_{0}=51\right)$. and $N_{b}$ is the total number of lattice sites within this circle. The dynamic order parameter of breathing transition is defined as $Q_{b}=\frac{\omega}{2 \pi} \oint m_{b}(t) d t$. The fluctuations in dynamic order (for breathing) $<\delta Q_{b}{ }^{2}>=<Q_{b}{ }^{2}>-<$ $Q_{b}>^{2}$. The average value of $Q_{b}$ and the fluctuation are calculated over $n_{c}=1000$ number of random samples. It is also checked that this number of samples is sufficient to have the data within the specified accuracy. The Fourier component $\hat{m}_{b}(\omega)=\frac{\omega_{0}}{2 \pi n_{c}} \int_{0}^{\frac{2 \pi n_{c}}{\omega_{0}}} m_{b}(t) e^{i \omega t} d t$. The integrated power for breathing $P_{b}=\int_{\frac{\omega_{0}}{2}}^{\frac{3 \omega_{0}}{2}} \hat{m}_{b}(\omega) d \omega$. In general, $\hat{m}_{b}(\omega)$ and $P_{b}$ are complex.

The quantities to study the spreading transition are: The boundary touching ring magnetisation density $m_{s}(t)=\sum s(x, y, t) / N_{s}$, where the summation is carried over the sites residing within the ring of width $\lambda / 2$ touching 
the boundary, and $N_{s}$ is the total number of lattice sites within that ring. It is a region bounded between two concentric $\left(x_{0}=51, y_{0}=51\right)$ circles having outer radius $r_{\text {out }}=L / 2$ and the inner radius $r_{\text {in }}=L / 2-\lambda / 2$. The dynamic order parameter of spreading transition $Q_{s}=\frac{\omega}{2 \pi} \oint m_{s}(t) d t$. The fluctuation in dynamic order (for spreading) $<\delta Q_{s}{ }^{2}>=<Q_{s}{ }^{2}>-<Q_{s}>^{2}$. The Fourier component $\hat{m}_{s}(\omega)=\frac{\omega_{0}}{2 \pi n_{c}} \int_{0}^{\frac{2 \pi n_{c}}{\omega_{0}}} m_{s}(t) e^{i \omega t} d t$. The integrated power for spreading $P_{s}=\int_{\frac{\omega_{0}}{2}}^{\frac{3 \omega_{0}}{2}} \hat{m}_{s}(\omega) d \omega$. Here also, $\hat{m}_{s}(\omega)$ and $P_{s}$ are complex, in general.

\section{Simulational results:}

A square lattice of size $L=101$ is considered. At the centre $\left(x_{0}=51, y_{0}=\right.$ $51)$ there is a source of spherical electromagnetic wave propagating radially outward. The initial configuration of the system was taken where all the Ising spins, $s(x, y, t=0$ ), assume the value +1 . The system is heated (slowly in the step $\Delta T=0.05)$ in presence of this field. For low enough temperature, i.e. $T=0.3$ (and $h_{0}=2.5, f_{0}=0.01, \lambda=15.0$ ) it is observed that no spin flip occurs and a dynamically pinned phase is observed (Fig-1(a)). On the other hand, at the temperature $T=1.45$,(keeping all the other parameter fixed), considerable number of flipping of the spins starts to occur near the centre, since the values of the magnetic fields are higher near the centre. Fig-1(b), shows snapshot of a typical breathing mode, where the local magnetisation $m_{b}(t)$ starts to oscillate.

The dynamics of a typical breathing mode is pictorially represented by Fig-2. Here, the snapshots at two different times are shown for a fixed set of values of $T=1.25, h_{0}=2.5, f=0.01$ and $\lambda=15.0$. Fig-2(a), shows the spin configuration at instant $t=3970$ MCSS and Fig-2(b) shows the same in a later instant $t=4000$ MCSS. From this figure, the dynamical breathing mode, is evident. A central and localised dynamical mode observed here, is called the breathing mode.

To distinguish the dynamical breathing mode from the dynamical spreading mode, a typical high temperature and high field dynamical mode is shown in Fig-3. For $T=2.30$ and $h_{0}=10.0$, the spin wave propagation is shown and it represents a propagation of spherical spin waves which spreads through the entire lattice. This is certainly a nonlocalised or spreading dynamical mode 
remarkably distinct from the localised or breathing mode.

As a result, in the breathing mode the instantaneous local magnetisation density $m_{b}(t)$ becomes a periodic function of time but oscillates asymmetrically about a nonzero avarage value. One such plot of $m_{b}(t)$ versus $t$ is shown in Fig-4(a) for the values of the parameter $T=0.5, h_{0}=2.5$. Keeping the value of $h_{0}=2.5$ fixed, the symmetric oscillation of $m_{b}(t)$ is observed at higher temperature $(T=1.25)$ and shown in Fig-4(b). One gets a transition, called the breathing transition, from low temperature dynamically symmetry broken phase to a high temperature dynamically symmetric phase.

Now, let us see what happens in the case of spin wave spreading mode. Whether the spin wave spreads over the entire lattice or not, it must be confirmed by studying the time variation of the magnetisation density within the ring (of finite width) touching the boundary of the lattice. This promted to define the ring magnetisation density in the way described in the last section (section-II). Here also, the boundary touching ring magnetisation density $m_{s}(t)$ is plotted against the time $t$ and shown in Fig-5. For $h_{0}=$ 2.5 and $T=2.0$ the symmetry broken oscillation of $m_{s}(t)$ is observed and shown in Fig-5(a). Increasing the temperature to $T=2.5$ (keeping $h_{0}=$ 2.5 fixed), the oscillation of $m_{s}(t)$ becomes symmetric (about $m_{s}(t)=0$ line). Here also, one gets a transition, called the spreading transition, from low temperature dynamically symmetry broken phase to a high temperature dynamically symmetric phase.

From the plots of $m_{b}(t)$ (in Fig-4) and $m_{s}(t)$ (in Fig-5), the high temperature symmetric and low temperature symmetry broken modes of oscillation were observed. These oscillations are periodic in nature. This periodicity is confirmed by studying the Fourier components $\hat{m}_{b}(\omega)$ and $\hat{m}_{s}(\omega)$ as the functions of $\omega$. These are called the power spectra of $m_{b}(t)$ and $m_{s}(t)$. The power spectra for the breathing transition is shown in Fig-6 and that for spreading transition is shown in Fig-7. From the figures, it is clear that the spin wave breathing and spreading modes are periodic (with the same periodicity,i.e., $f=0.01$ of the spherical electromagnetic wave) in both (symmetric and symmetry-broken) phases.

To study the symmetry broken dynamic phase transitions (both breathing and spreading) quantitatively and to estimate the transition temperatures precisely, one has to define the order-parameters for the transitions. The time averaged magnetisation density over a full cycle of the EM field defines the dynamic order parameter. By definition, the symmetry broken phase will 
yield nonzero value of the dynamic order parameter and hence represent the dynamically ordered phase. The disordered phase (vanishingly small value of the order parameter) is dynamically symmetric phase. The temperature dependence of the dynamic order parameters $Q_{b}$ (for breathing) and $Q_{s}$ (for spreading) are plotted in Fig- 8 for two different values of $h_{0}$ (equals to 2.5 in Fig-8(a) and equals to 3.5 in Fig-8(b)).

To have a precise measure of the dynamic breathing and spreading transitions the variances $\left\langle\left(\delta Q_{b}\right)^{2}>\right.$ and $<\left(\delta Q_{s}\right)^{2}>$ of $Q_{b}$ and $Q_{s}$ respectively are studied as the function of temperature and shown in Fig-9. Here the peaks of $<\left(\delta Q_{b}\right)^{2}>$ and $<\left(\delta Q_{s}\right)^{2}>$ indicate the respective transitions. In Fig-9(a), the plots are shown for $h_{0}=2.5$. The breathing and spreading transitions occur at $T_{b}=0.95$ and $T_{s}=2.25$ respectively. Similar plots for $h_{0}=3.5$ are shown in Fig-9(b). In this case, the breathing and spreading transitions occur at $T_{b}=0.75$ and $T_{s}=2.20$ respectively. It may be mentioned at here that the small step of temperature, by which the system was heated from a perfectly ordered configuration, is $\Delta T=0.05$. So, the transition temperature has the maximum error of amount \pm 0.05 .

The absolute value of integrated powers, (obtained from power spectra) for breathing and spreading modes, are studied also as a function of temperature and shown in Fig-10. The integrated powers $\left|P_{b}\right|$ for breathing mode and $\left|P_{s}\right|$ for spreading mode are plotted against the temperature $T$ in Fig-10(a) for $h_{0}=2.5$ and in Fig-10(b) for $h_{0}=3.5$ respectively. From the figures it is clear that the breathing transition is also indicated approximately by the sharp peak of $\left|P_{b}\right|$. However, the integrated power $\left|P_{s}\right|$ for the spreading transition shows a relatively smeared peak above the spreading transition temperature $T_{s}$.

Obtaining the breathing and spreading transition temperatures for different values of the amplitude $\left(h_{0}\right)$ of the spherical EM field, a comprehensive phase diagram is drawn in the plane formed by $h_{0}$ and $T$. This phase diagram is shown in Fig-11. Three distinct phases, the pinned, the breathing and the spreading and their boundaries are shown (with maximum errors in measuring the transition temperatures). The boundaries representing the breathing and spreading transitions come closer as $h_{0}$ decreases and eventually reach the limit $T=2.269 \ldots$ (the Onsager's value) as $h_{0} \rightarrow 0$. 


\section{Concluding remarks:}

The dynamical responses of two dimensional Ising ferromagnet irradiated by a spherical electromagnetic wave are studied by Monte Carlo simulation. For very low temperature and small values of the amplitude of EM wave, the system remains in a pinned state. As the system is heated the spins in the central regions starts to flip and a very localised dynamical breathing mode was observed. The local magnetisation density starts to oscillates. A dynamical phase transition, called breathing transition associated with a symmetry breaking is observed. For high value of the temperature of the system the collective spin wave (spherically symmetric) starts to propagate and spreads over the entire lattice. A dynamical spreading mode was observed. Here also a dynamic symmetry breaking spreading transition is observed. These breathing and spreading transition temperatures depend on the amplitude of the EM wave. A comprehensive phase diagram is drawn in the plane formed by the temperature and the amplitude of the EM wave. The boundaries of breathing and spreading transitions come closer as the amplitude of the EM wave decreases and eventually merge at the equilibrium ferro-para transition point for the two dimensional Ising ferromagnet as the value of the amplitude approaches zero.

The dynamic symmetry breaking breathing and spreading transitions are new kinds of nonequilibrium phase transitions observed in two dimensional ising ferromagnet irradiated by spherical electromagnetic wave. There are further scopes of studies in this front. One may study it in three dimensions also. The detail study of this phase transition and scaling analysis would lead to have an idea about the universality class of these transitions. It would be interesting and supportive to study these transitions experimentally using ultrathin Co film irradiated by strong EM wave by surface magneto optic Kerr effect.

Acknowledgements: Author would like to thank the library facilities provided by the University of Calcutta. 


\section{References}

1. M. Acharyya, Int. J. Mod. Phys. C, 16 (2005) 1631, See also, B. K. Chakrabarti, M. Acharyya, Rev. Mod. Phys., 71 (1999) 847,

2. T. Tome and M. J. de Oliveira, Phys. Rev. A 41 (1990) 4251

3. M. Acharyya, Phys. Rev. E 56 (1997) 2407

4. M. Acharyya, Phys. Rev. E, 56 (1997) 1234

5. M. Acharyya, Phys. Rev. E, 58 (1998) 174

6. M. Acharyya, Phys. Rev. E, 58 (1998) 179

7. M. Acharyya, Phys. Rev. E, 59 (1999) 218

8. S. W. Sides, P. A. Rikvold and M. A. Novotny, , Phys. Rev. Lett. 81 (1998) 834

9. G. Korniss, C. J. White, P. A. Rikvold and M. A. Novotny, Phys. Rev. E, 63 (2001) 016120

10. G. Korniss, P. A. Rikvold, M. A. Novotny, Phys. Rev. E, 66 (2002) 056127

11. G. M. Buendia and P. A. Rikvold, Phys. Rev. E, 78 (2008) 051108

12. H. Park and M. Pleimling, Phys Rev Lett 109 (2012) 175703.

13. G. Gulpinar, D. Demirhan, M. Buyukkilic, Phys. Lett. A 373 (2009) 511; B. Deviren, M. Keskin,Phys. Lett. A 374 (2010) 3119. M. Keskin, O. Canko, M. Kirak, Phys. Stat. Solidi B, 244 (2007) 3775;

14. M. Acharyya, J. Magn. Magn. Mater. 323 (2011) 2872

15. E. Vatansever and H. Polat, J. Magn. Magn. Mater., 343 (2013) 221

16. M. Keskin, O. Canko, B. Deviren, Phys. Rev. E 74 (2006) 011110

17. U. Temizer, E. Kantar, M. Keskin, O. Canko, J. Magn. Magn. Mater. 320 (2008) 1787 
18. M. Acharyya, Int. J. Mod. Phys. C 14 (2003) 49.

19. H. Jung, M. J. Grimson, C. K. Hall, Phys Rev B 67 (2003) 094411.

20. H. Jung, M. J. Grimson, C. K. Hall, Phys Rev E 68 (2003) 046115.

21. M. Acharyya, Phys. Rev. E. 69 (2004) 027105

22. O. Jiang, H. N. Yang, G. C. Wang, Phys Rev B 52 (1995) 14911; Q. Jiang, H. N. Yang and G. C. Wang, J. Appl. Phys. 79 (1996) 5122.

23. M. Acharyya, Physica Scripta, 84 (2011) 035009

24. M. Acharyya, J. Magn. Magn. Mater., 334 (2013) 11

25. M. Acharyya, Preprint (under review), cond-mat-arXiv:1301.3071

26. K. Binder and D. W. Heermann, 1997, Monte Carlo Simulation in Statistical Physics (Springer Series in Solid State Sciences) (New York: Springer) 

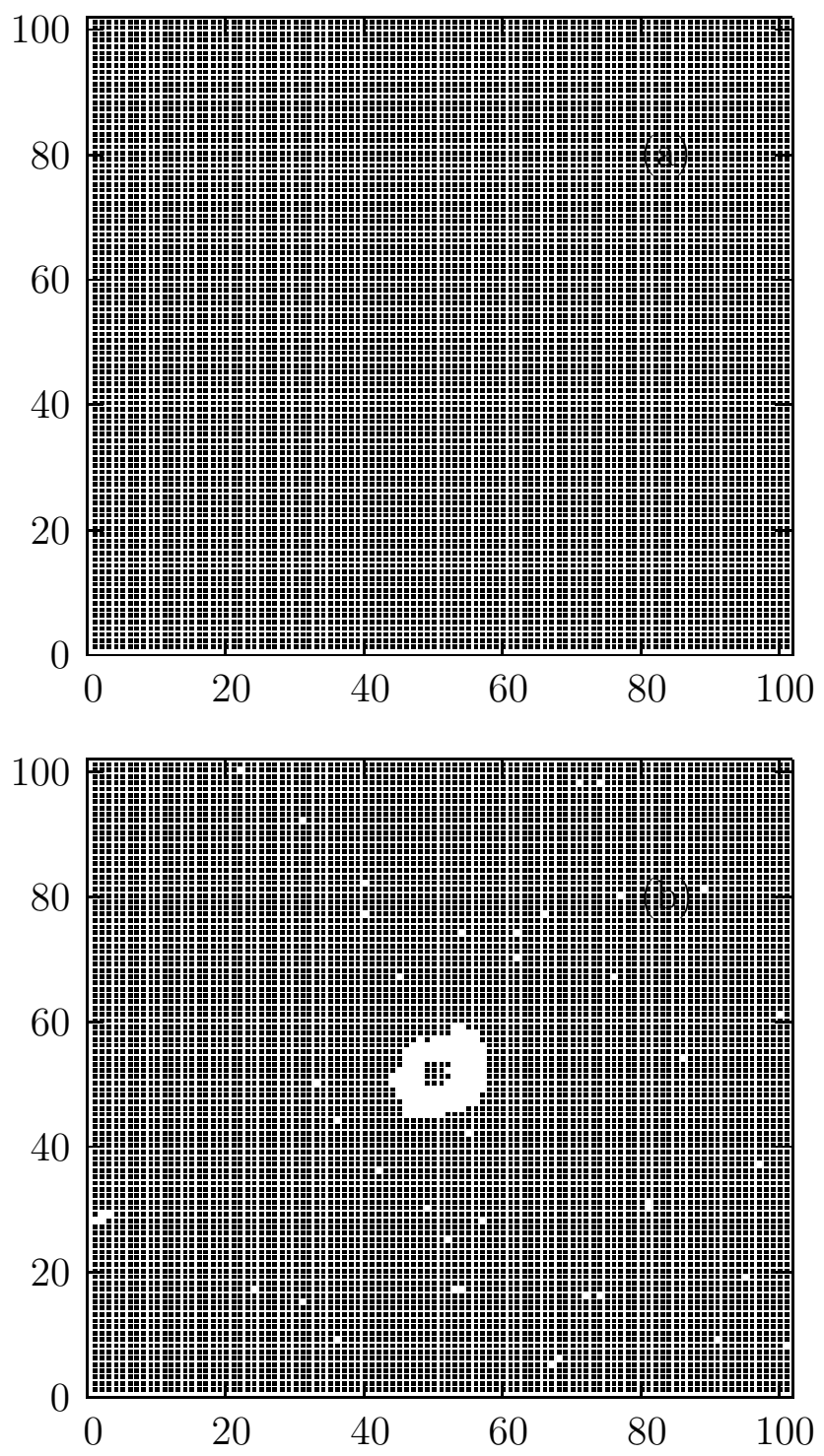

Fig-1. The pinned (a) and breathing (b) modes on the lattice. Dots represents up spins only. (a) $h_{0}=2.5, T=0.30$ and (b) $h_{0}=2.5$ and $T=1.45$. Here, $\lambda=15.0, f_{0}=\frac{\omega_{0}}{2 \pi}=0.01$ and $t=4000 \mathrm{MCSS}$. 

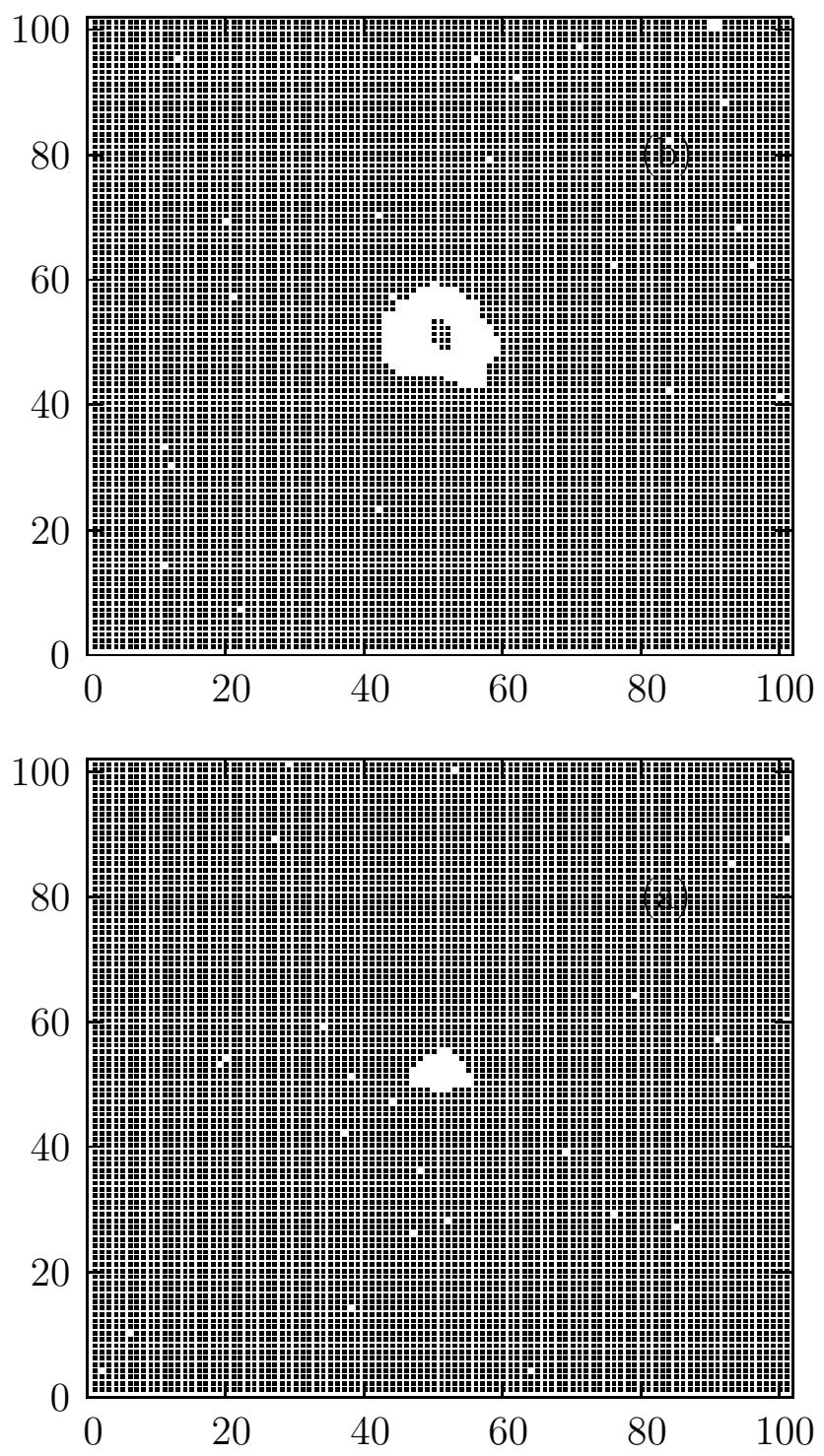

Fig-2. The breathing mode on the lattice. (a) $h_{0}=2.5, f_{0}=0.01, \lambda=15.0$, $T=1.25$ at $t=3970$ MCSS. (b) $h_{0}=2.5, f_{0}=0.01, \lambda=15.0, T=1.25$ at $t=4000 \mathrm{MCSS}$. 

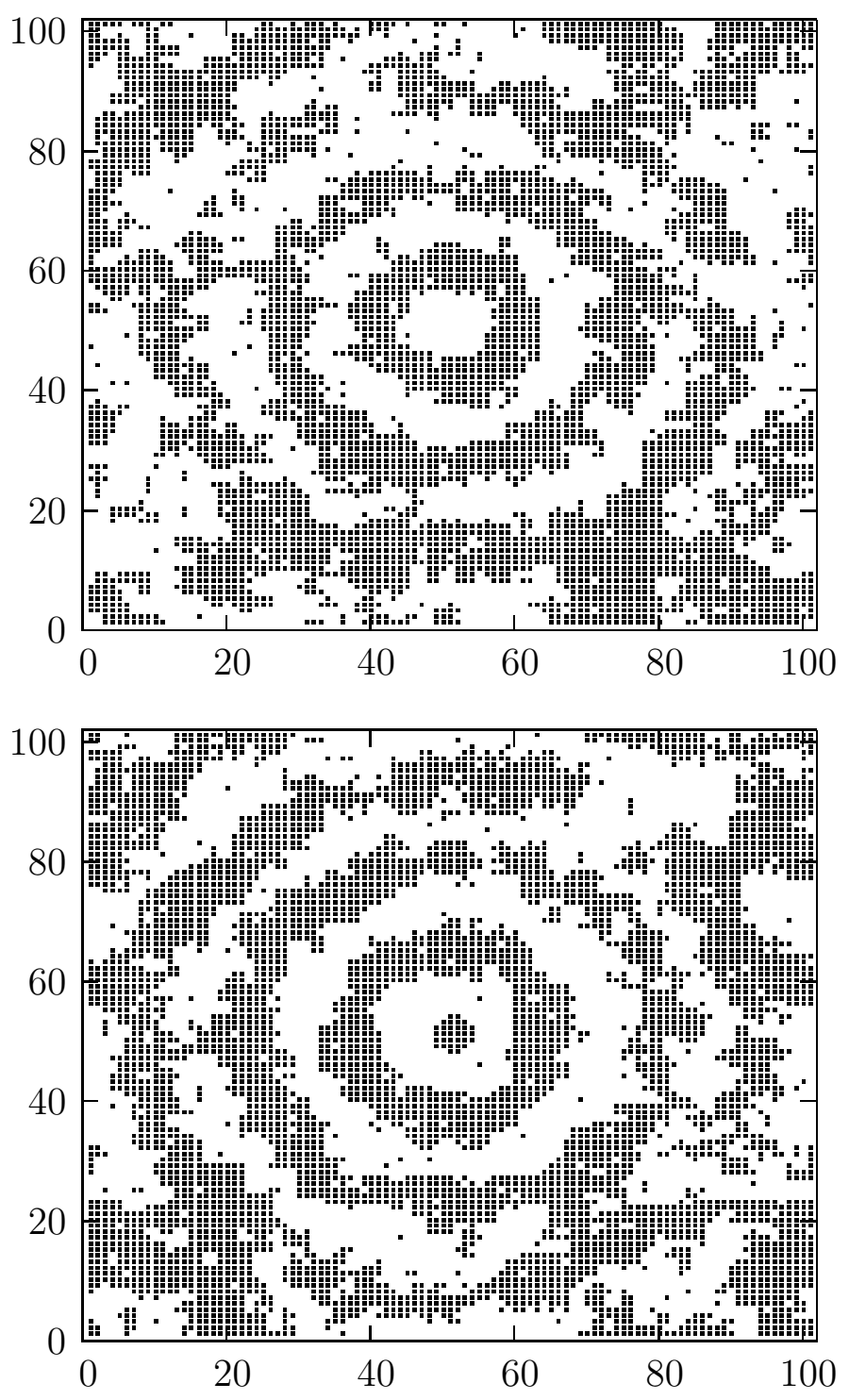

Fig-3. A high field $\left(h_{0}=10.0\right)$-high temperature $(T=2.30)$ spin wave propagation mode. The up spins $\left(S_{i}=+1\right)$ are shown by black dot. The bottom one shows a spin configuration at 3970 MCSS and the top one shows that at 4000 MCSS. Here, $f_{0}=0.01$ and $\lambda=15.0$. 

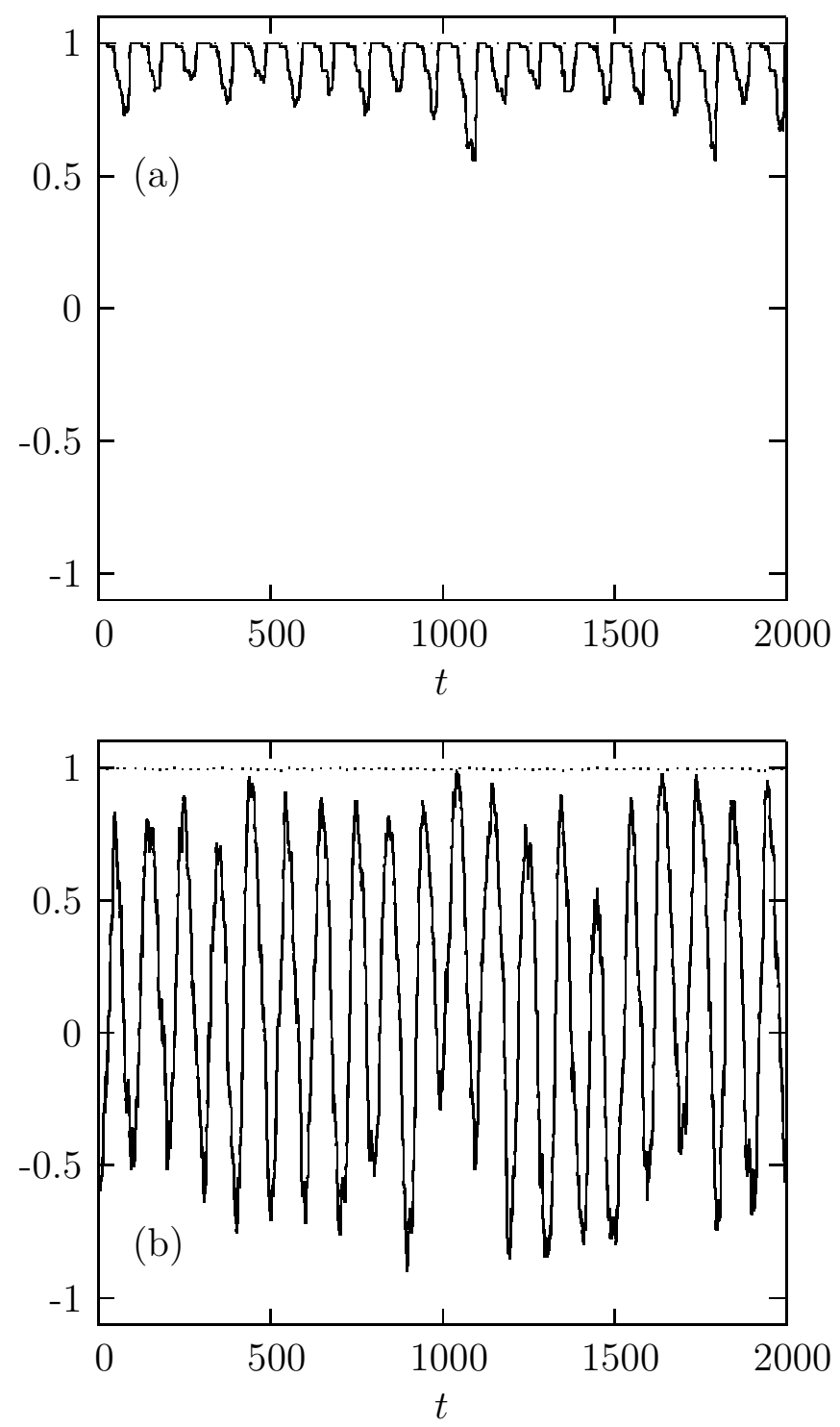

Fig-4. $m_{b}(t)$ (solid line) and $m_{s}(t)$ (dotted line) are plotted against time $t$. (a) $h_{0}=2.5, \lambda=15.0$ and $T=0.5$ and (b) $h_{0}=2.5, \lambda=15.0$ and $T=1.25$. 

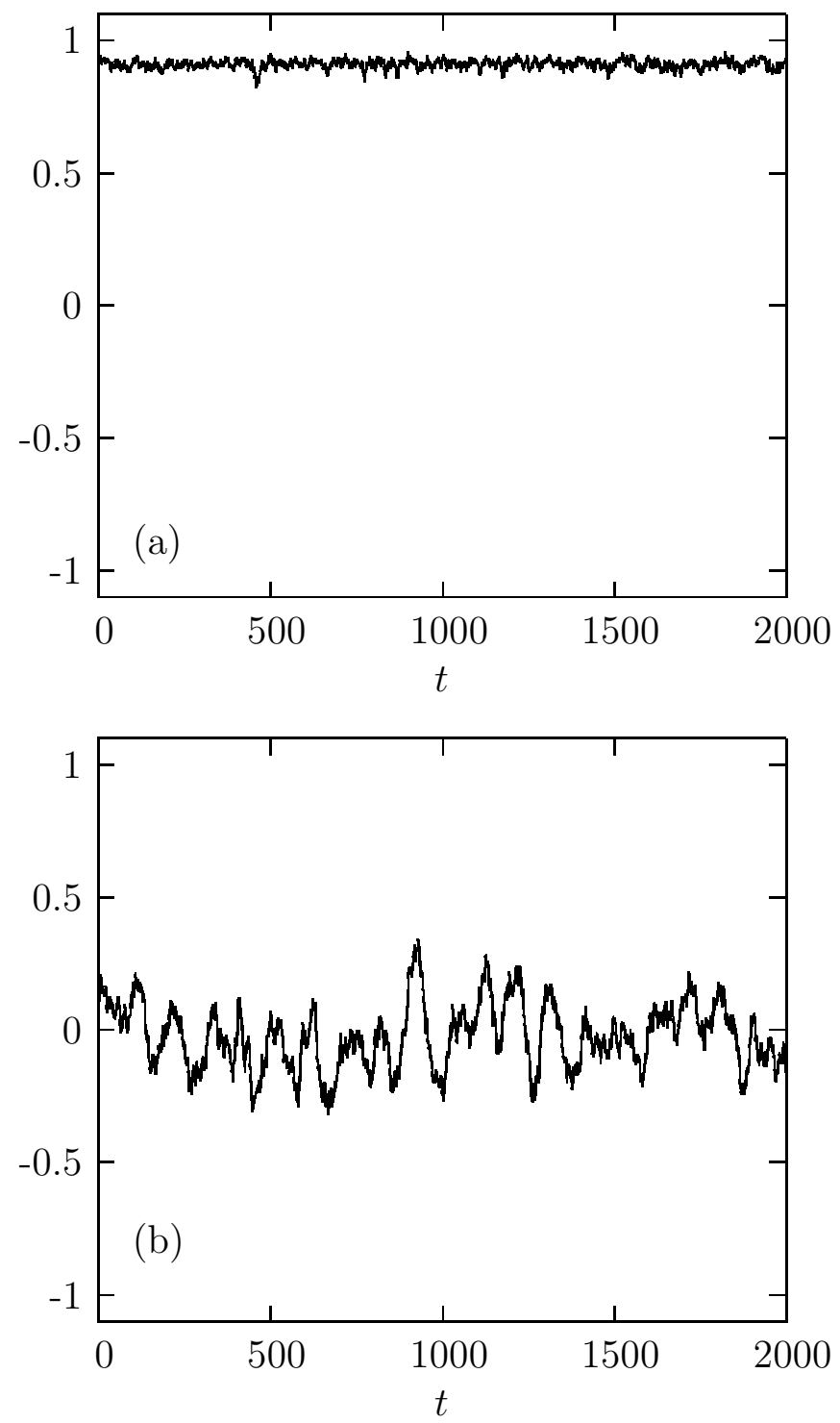

Fig-5. $m_{s}(t)$ is plotted against time $t$. (a) $h_{0}=2.5, \lambda=15.0$ and $T=2.0$ and (b) $h_{0}=2.5, \lambda=15.0$ and $T=2.5$. 

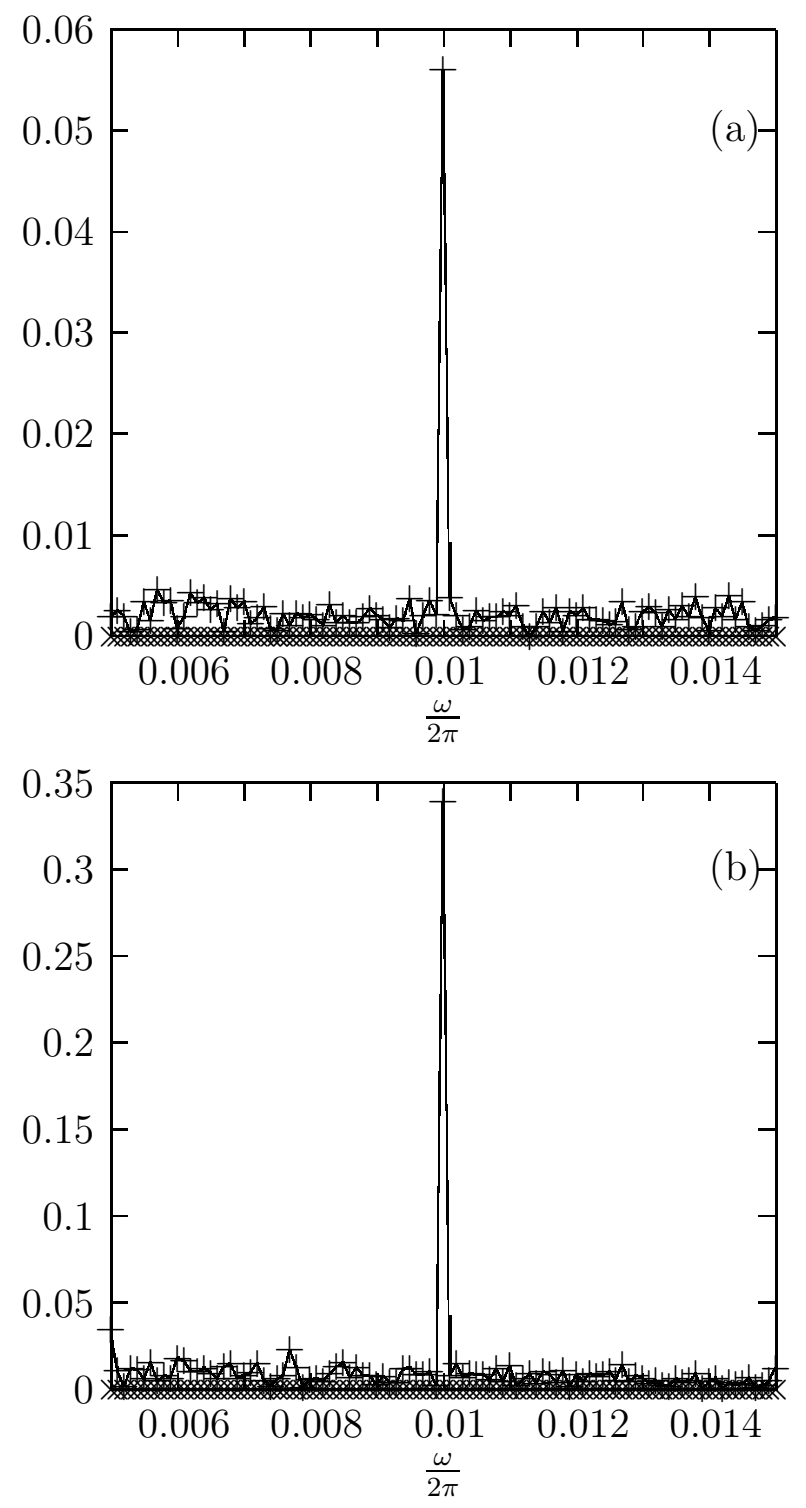

Fig-6. The power spectra for breathing transition. $\left|\hat{m}_{b}(\omega)\right|(+)$ and $\left|\hat{m}_{s}(\omega)\right|(\times)$ plotted against $\frac{\omega}{2 \pi}$, for (a) $h_{0}=2.5, \lambda=15.0$ and $T=0.5$ and (b) $h_{0}=2.5$, $\lambda=15.0$ and $T=1.25$. 

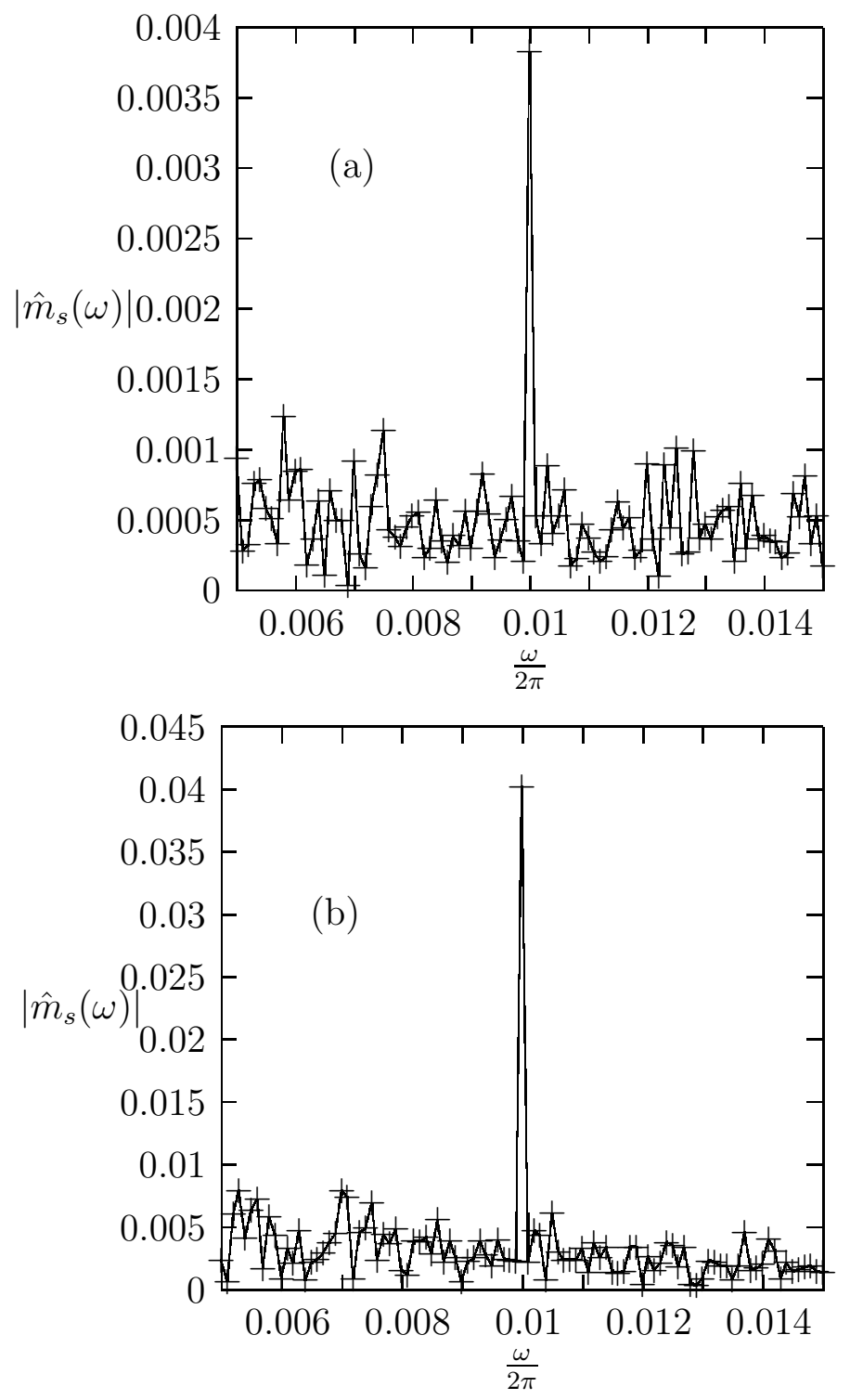

Fig-7. The power spectra for spreading transition. for (a) $h_{0}=2.5, \lambda=15.0$ and $T=2.0$ and (b) $h_{0}=2.5, \lambda=15.0$ and $T=2.5$. 

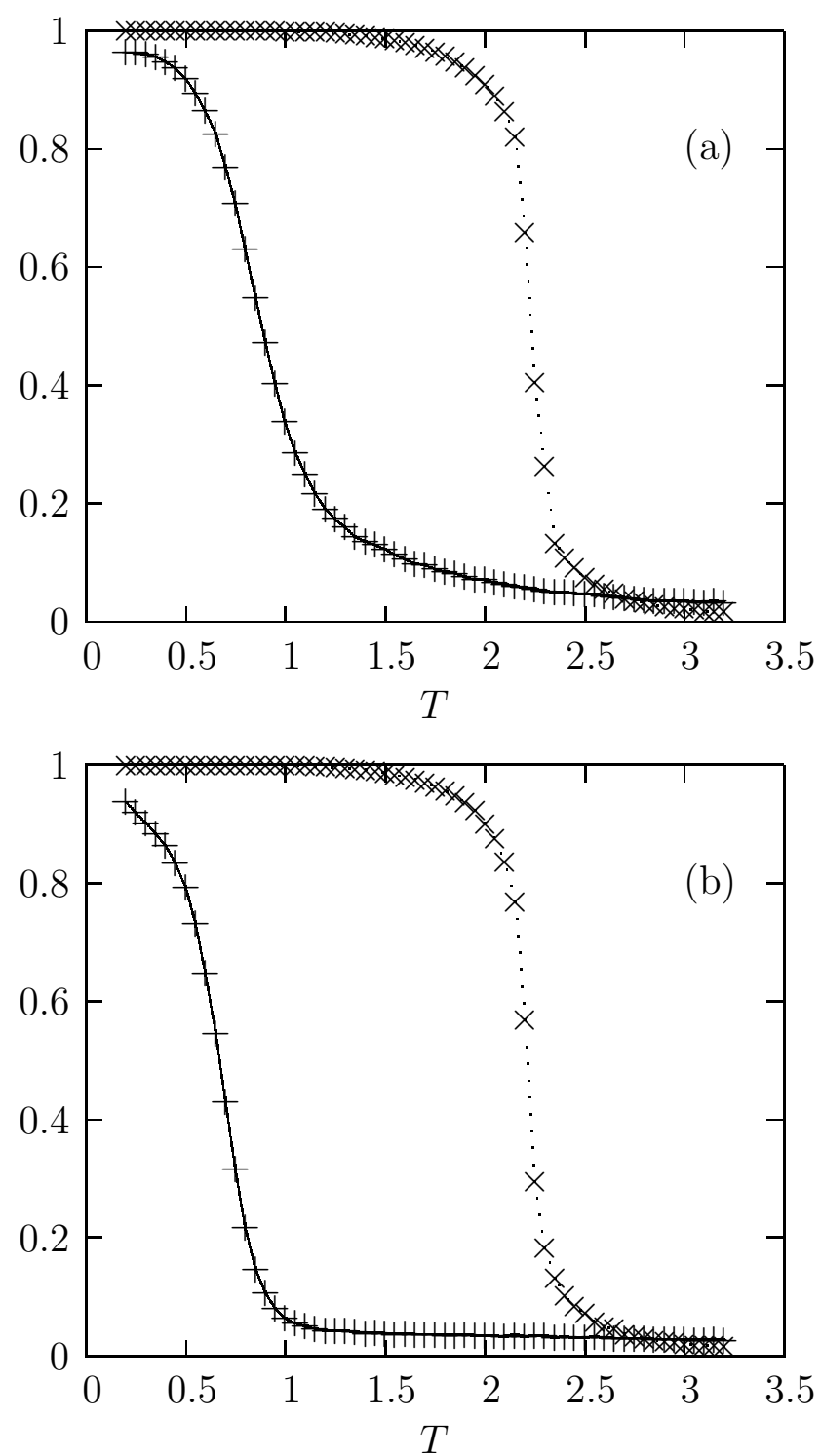

Fig-8. The plots of $Q_{b}(+)$ and $Q_{s}(\times)$ as a function of temperature $T$. (a) $h_{0}=2.5, f=0.01$ and $\lambda=15.0$ and (b) $h_{0}=3.5, f=0.01$ and $\lambda=15.0$. 

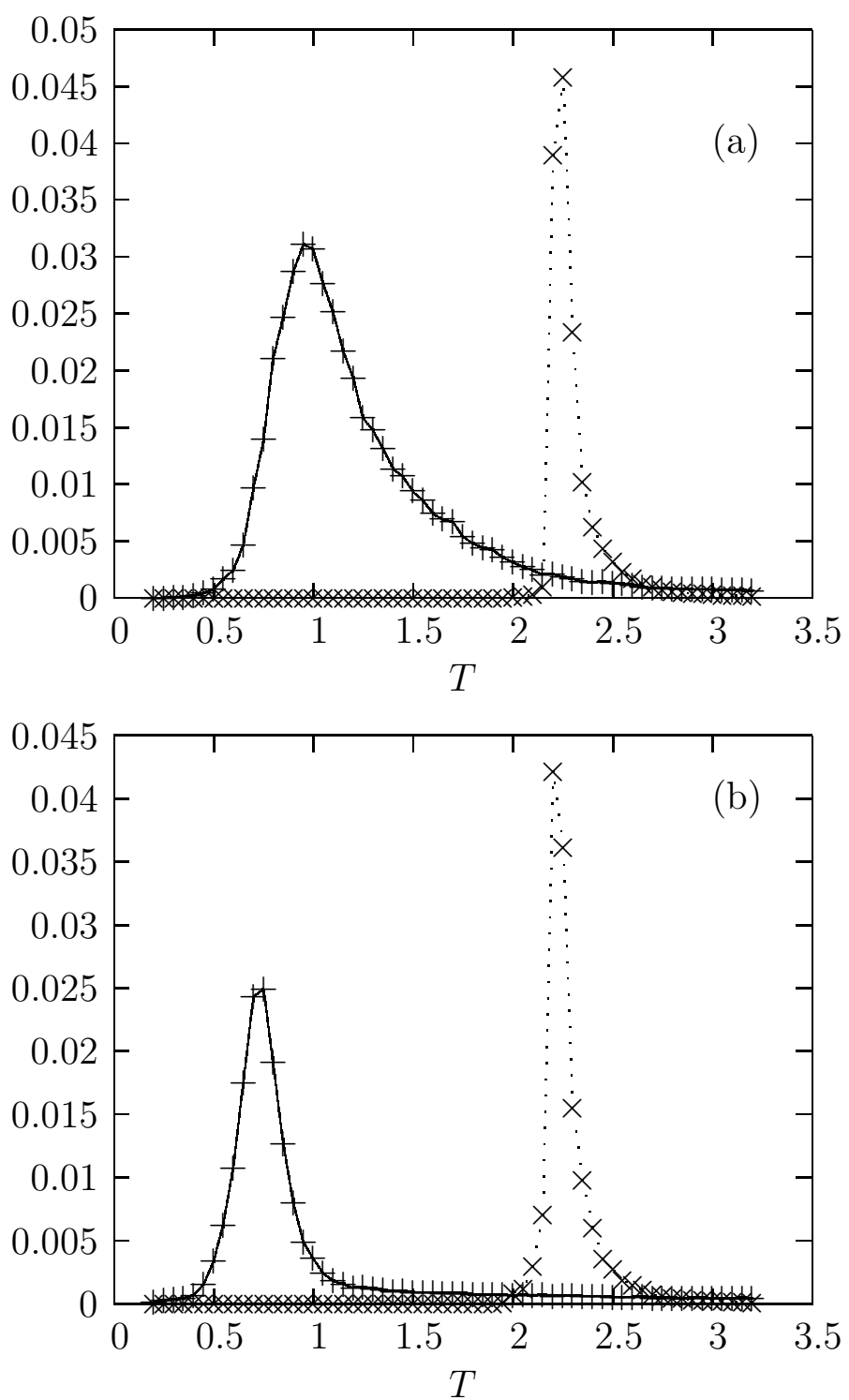

Fig-9. Fluctuations of $Q_{b}(+)$ and $Q_{s}(\times)$ plotted against temperature $(T)$ for two different values of field amplitude $\left(h_{0}\right)$ of propagating field. (a) $h_{0}=2.5$ and (b) $h_{0}=3.5$. Here, $\lambda=15.0$ and $f=0.01$. 

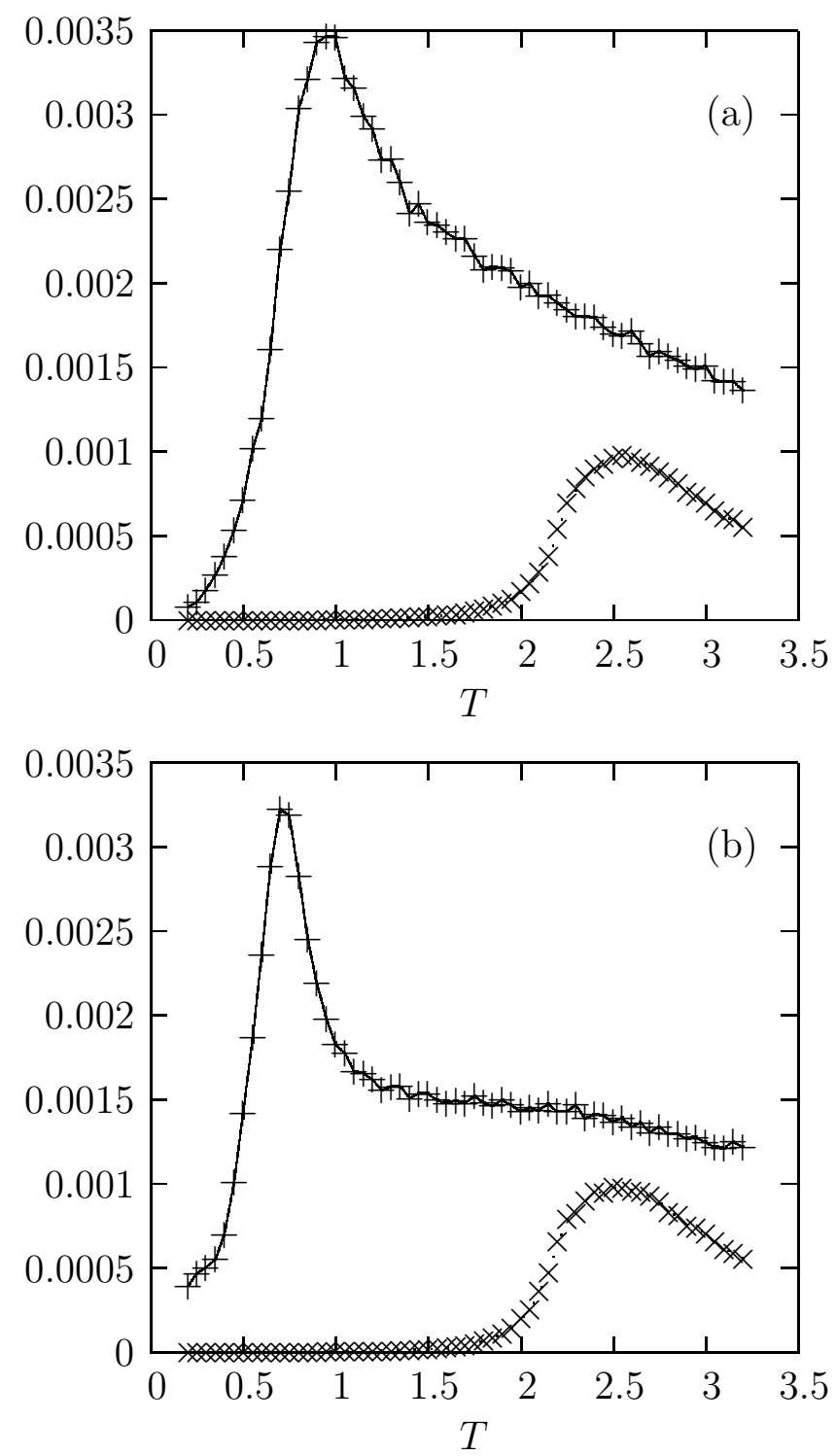

Fig-10. The plots of integrated powers, i.e., $\left|P_{b}\right|(+$, for breathing $)$ and $\left|P_{s}\right|\left(\times\right.$, for spreading) as functions of temperature (T). (a) $h_{0}=2.5$ and (b) $h_{0}=3.5$. 


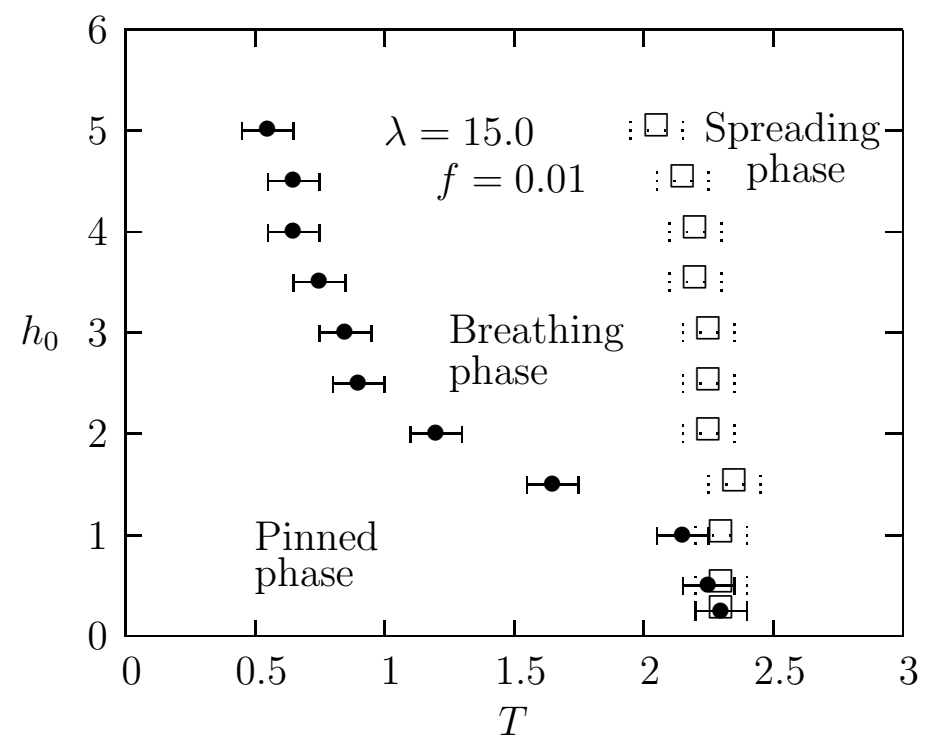

Fig-11. The phase diagram for symmetry breaking dynamic breathing and spreading transitions. Here, $f_{0}=0.01$ and $\lambda=15.0$. 